\title{
Assessing methods of identifying open water bodies using Landsat 8 OLI imagery
}

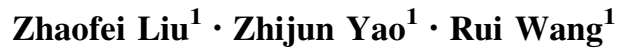

Received: 18 January 2015/Accepted: 29 April 2016/Published online: 11 May 2016

(C) Springer-Verlag Berlin Heidelberg 2016

\begin{abstract}
Remote sensing is an effective technology for monitoring water resources. However, many methods in remote sensing imagery used to identify open bodies of water have often been shown to produce varying water body classification results for the same bodies of water. Therefore, it is necessary to have measures to assess these methods. In this study, we used datasets collected in the field to assess methods for identifying open water bodies using images from the Landsat 8 Operational Land Imager. From this, we clarified the difference in the performance between the use of spectral reflectance images and that of digital number (DN) value images for the classification of water bodies. The results showed that the normalized difference water index (NDWI), calculated using green and near-infrared bands $\left(\mathrm{NDWI}_{\mathrm{Green} / \mathrm{NIR}}\right)$ with reflectance, captured correct control points with an accuracy of greater than $95 \%$ and was therefore the superior method. The result of a comparison in performance in terms of the NDWI between reflectance images and DN value images was consistent with their initial definitions. The NDWI indices calculated by the initial definitions yielded more reasonable results in the classification of water bodies. The optimized threshold, calibrated and validated by 737 field control points, generated water classification results with a higher confidence in this study. We think that it might be better to set the optimized threshold of $\mathrm{NDWI}_{\mathrm{Green} / \mathrm{NIR}}$ to
\end{abstract}

Zhaofei Liu

zfliu@igsnrr.ac.cn

$\triangle$ Zhijun Yao

yaozj@igsnrr.ac.cn

1 Institute of Geographic Sciences and Natural Resources Research, Chinese Academy of Sciences, Beijing 100101, China
-0.05 instead of the value of zero used in many studies. However, more optimized thresholds for other regions need to be calibrated and confirmed if data are available. Our results indicated that NDWI methods are more suitable for water body classification than single-band methods when the frequency histogram method is used.

Keywords Remote sensing imagery · Normalized difference water index (NDWI) - Water body extraction . Optimized threshold $\cdot$ Tibetan plateau $\cdot$ Spectral reflectance

\section{Introduction}

Water is a necessary resource that forms the basis of all life. Accurate monitoring and assessment of water resources are necessary for sustained water resource management. Remote sensing technology is considered a time- and cost-effective approach for monitoring large-scale water resources (Okin et al. 2001).

Several methods can be used to identify open water bodies from remote sensing data. Open water bodies can be identified from images based on land cover classification methods, such as maximum likelihood classification (Paola and Schowengerdt 1995), decision tree classification, support vector machine classification, artificial neural network classification (Hepner et al. 1990), and others. However, because the spectral reflectance of water is considerably lower than those of other land surface materials (Smith 2012a, b), it is easier to identify bodies of water than other land cover types in remote sensing images. Therefore, water body extraction methods usually apply image segmentation algorithms (threshold selection) using a single spectral band or the Normalized Difference Water Index (NDWI) calculated from multiple bands. In other words, 
images can be classified as water or non-water by identifying a threshold value.

Several studies have used threshold selection in a single band from Landsat Thematic Mapper (TM) imagery. TM bands 4,5 , and 7 are typically used because they are absorbed to a greater extent by water and are reflected more strongly by vegetation and bare soil, than other bands. Frazier and Page (2000) evaluated water boundary mapping using different TM bands. They pointed out that Landsat TM data can be used to accurately map water bodies. They found that TM band 5 exhibited the best performance, whereas bands 4 and 7 yielded lower accuracy. Braud and Feng (1998) also found that thresholding by using TM band 5 was the most reliable method for identifying water bodies. Manavalan et al. (1993) successfully identified open water bodies by applying density slicing to TM band 4. Moller-Jensen (1990) identified water pixels from TM bands 4 and 5 by image threshold segmentation.

NDWI uses multispectral bands to enhance water features and eliminate noise components and has been widely applied to identify water bodies in various regions. The method mainly comprises three NDWIs- NDWI $_{\text {Green/NIR }}$

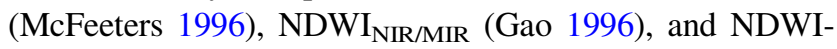
Green/MIR (Xu 2006) - that are calculated by [(Green $\mathrm{NIR}) /($ Green $+\mathrm{NIR})],[(\mathrm{NIR}-\mathrm{MIR}) /(\mathrm{NIR}+\mathrm{MIR})]$, and $[($ Green $-\mathrm{MIR}) /($ Green $+\mathrm{MIR})], \quad$ respectively. NDWIGreen/MIR is identical to the Normalized Difference Snow Index (NDSI) (Dozier 1984), which has been widely applied to distinguish snow from other land cover (Dozier 1989). $\mathrm{NDWI}_{\text {Green/NIR }}$ and NDWI $\mathrm{NIR}_{\text {/MIR }}$ used images with spectral reflectance to identify water features when they were initially developed, whereas $\mathrm{NDWI}_{\text {Green/MIR }}$ used images with digital number (DN) values.

Many studies successfully have applied $\mathrm{NDWI}_{\text {Green/MIR }}$ to identify open water bodies while using images with spectral reflectance (e.g., Campos et al. 2012; Lu et al. 2013; Ma et al. 2007; Sun et al. 2012; Li et al. 2013). $\mathrm{NDWI}_{\text {Green/NIR }}$ and $\mathrm{NDWI}_{\text {Green/MIR }}$ were initially developed to identify open water bodies, whereas $\mathrm{NDWI}_{\mathrm{NIR} / \mathrm{MIR}}$ was developed to identify vegetation waters (Leauthaud et al. 2013). Moreover, some studies achieved satisfactory results by applying $\mathrm{NDWI}_{\mathrm{NIR} / \mathrm{MIR}}$ to identify open water bodies (Campos et al. 2012; Ma et al. 2007; de Alwis et al. 2007; and others).

Threshold selection is a key step in identifying water bodies from single-band or NDWI images. McFeeters (1996) and $\mathrm{Xu}$ (2006) suggested that the threshold of $\mathrm{NDWI}_{\text {Green/NIR }}$ and $\mathrm{NDWI}_{\mathrm{NIR} / \mathrm{MIR}}$ can be set to zero. This can lead to overestimating or underestimating water area when using an arbitrary, empirically selected threshold because threshold values vary with region or image phase.
Threshold adjustment in certain situations can yield a more accurate delineation of water bodies (Ji et al. 2009). Frequency histograms have been used for threshold selection to extract water bodies from images ( $\mathrm{Li}$ and Sheng 2012; Ryu et al. 2002; Lacaux et al. 2007). The ideal histogram has a bimodal distribution where the two peaks represent the water peak and the non-water peak. Li et al. (2013) also used the maximum inter-class variance method (the Otsu method) to determine the NDWI threshold. However, it remains necessary to evaluate different methods with appropriate thresholds to determine the best one. Ji et al. (2009) suggested that data from actual situations are appropriate for such an evaluation.

In general, it is necessary to use field data to assess methods for identifying water bodies from remote sensing imagery. Some studies have suggested that a careful hand digitization method produces the highest accuracy in delineating water bodies (glaciers) from satellite imagery (Bishop et al. 2004; Raup et al. 2007). However, it is difficult to identify the boundaries of water bodies with the naked eye because water boundary pixels and adjoining non-water pixels usually possess similar colors in satellite imagery. Therefore, in this study, we used field data to evaluate the performance of different methods in identifying water bodies through remote sensing imagery.

As mentioned above, some studies to identify water bodies have used the DN value of images to calculate NDWIs, whereas others have used the reflectance of images. To determine whether the NDWI should be calculated using DN values or the reflectance of images, the performance of DN value-induced NDWIs and reflectance-induced NDWIs should be assessed.

Therefore, we evaluated NDWIs calculated by both the DN value and reflectance in this study. The Landsat 8 satellite, which includes the Operational Land Imager (OLI) and the Thermal Infrared Sensor (TIRS), was launched in February 2013. Although the satellite can provide more options for images when applying remote sensing imagery in related studies, there has been scant evaluation of methods for identifying water bodies from Landsat 8 OLI images.

The objective of this study is to evaluate methods for identifying open water bodies from Landsat 8 OLI imagery. We used randomly assigned field datasets to calibrate the optimal threshold for each image, including single bands with the DN value and spectral reflectance, and calculated the NDWIs of images. The optimized thresholds were then used to identify water bodies from each image. The performances of different methods were calibrated and tested using the randomly assigned field datasets, which included 100 independent datasets. Furthermore, the difference in pixel values between pure water and the pure soil adjoining water bodies was analyzed to test these 
classification methods. The performance of the frequency histogram method in the classification of water bodies was also evaluated by field datasets. Finally, we discuss threshold calibration, the difference in performance between reflectance and DN value images, and the assessment of methods for identifying water bodies from remote sensing imagery.

\section{Materials and methods}

\section{Study area}

Four lakes (Fig. 1) in the northern Tibetan plateau (also called Qiangtang), China, were selected for this study. Qiangtang is a vast highland area with many large lakes (most of China's lakes are located in this region). The area contains 786 natural lakes with areas of greater than $1 \mathrm{~km}^{2}$ (Dong et al. 2010). Because of extreme environmental conditions (high altitude, remoteness, and challenging climate), it is very difficult to travel in this scientifically important region. All four lakes chosen for our study are at an altitude of over $4880 \mathrm{~m}$, with a mean altitude of $5051 \mathrm{~m}$. The water quality and areas of the four lakes are shown in Table 1 .

\section{Dataset}

\section{Field data}

A total of 737 ground control points (GCP) for the boundaries of the four lakes were collected on October 24, 2013, and the satellite data were acquired on the same day.

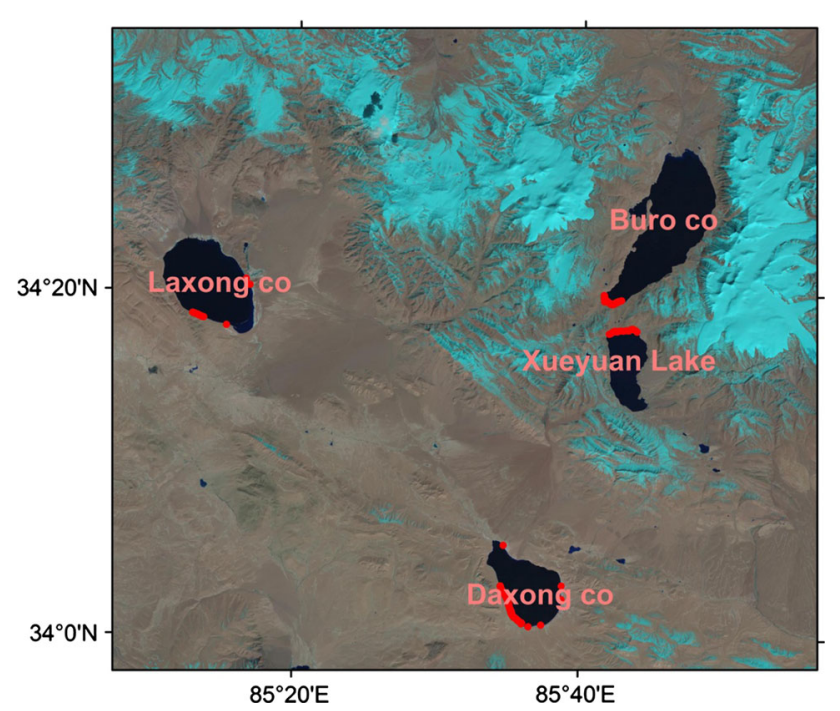

Fig. 1 Locations of four lakes (solid red dots represent ground control points)
The coordinates of the control points were collected using a Magellan Triton 400 handheld global positioning system (GPS) device. Its built-in signal augmentation reception provided an accuracy of within three meters.

\section{Remote sensing data}

Landsat 8 OLI imagery data were used in this study. The OLI sensor provides improved signal-to-noise $(\mathrm{SN})$ radiometric performance quantized over a 12-bit dynamic range. This translates into 4096 potential gray levels in an image, compared with only 256 gray levels produced by eight-bit instruments. Improved SN performance enables better characterization of the state and conditions of the land cover. The Landsat 8 OLI image data consist of nine spectral bands, with a spatial resolution of $30 \mathrm{~m}$ for bands 1-7 and band 9. The resolution of band 8 (panchromatic) is $15 \mathrm{~m}$ (The details of Landsat 8 products can be found at http://landsat7.usgs.gov/landsat8.php). The multispectral imagery of Landsat 8 OLI was obtained from http://glovis. usgs.gov/. An image (Path 142 and Row 036) on October 24, 2013, was acquired over the four lakes. Multispectral imagery, including bands $1-7$ and band 9 , was used in this study.

The correction and calibration of images to achieve a satisfactory representation of the Earth's surface is a fundamental consideration for all applications (Eastman 2001). The raw pixel values of images (known as digital numbers, or DN) were converted to true measures of reflective power (spectral radiance). The radiance image data items were then atmospherically corrected to obtain the spectral reflectance image. Details of this processing can be found in the Landsat 8 Science Data Users' Handbook (http://landsat7.usgs.gov/Landsat8_Using_Product. php). Finally, both reflectance and DN value images were used in our application.

\section{Methods}

\section{Water body extraction methods}

The three NDWIs used in this study $\left(\mathrm{NDWI}_{\text {Green/NIR }}\right.$, $\mathrm{NDWI}_{\text {Green/MIR }}$, and $\mathrm{NDWI}_{\mathrm{NIR} / \mathrm{MIR}}$ ) were calculated from the multispectral imagery of Landsat 8 OLI. "Green," "NIR" (near-infrared), and "MIR" (mid-infrared) identify Landsat 8 OLI bands 3, 5, and 6, respectively. Thus, $\mathrm{NDWI}_{\text {Green/NIR }}, \mathrm{NDWI}_{\text {Green/MIR }}$, and $\mathrm{NDWI}_{\mathrm{NIR} / \mathrm{MIR}}$ were referred to as NDWI35, NDWI36, and NDWI56, respectively. It needs to be noted that the wavelength of these three bands for Landsat 8 was in the ranges of the corresponding bands for Landsat 7 but with slight differences. The NDWIs of Landsat 8 OLI were calculated as follows: 
Table 1 Water quality and area of four lakes

\begin{tabular}{lcccc}
\hline & Laxong co & Daxong co & Buro co & Xueyuan lake \\
\hline Altitude $(\mathrm{m})$ & 4904.8 & 4893.1 & 5181.7 & 5225.0 \\
Temperature $\left({ }^{\circ} \mathrm{C}\right)$ & 4.0 & 4.5 & 2.1 & 3.7 \\
pH & 9.8 & 9.3 & 9.4 & 9.4 \\
Dissolved oxygen $(\mathrm{mg} / \mathrm{L})$ & 5.9 & 6.1 & 6.6 & 6.4 \\
Salinity $(\mathrm{ppt})$ & 8.6 & 12.2 & 5.2 & 1.7 \\
Redox potential $(\mathrm{ms} / \mathrm{cm})$ & 47.6 & 18.8 & 61.9 & 28.0 \\
Conductivity $(\mathrm{ms} / \mathrm{cm})$ & 12.7 & 16.7 & 8.2 & 2.9 \\
Total dissolved solids $(\mathrm{g} / \mathrm{L})$ & 7.9 & 10.4 & 5.1 & 1.8 \\
Seawater gravity $(\sigma \mathrm{t})$ & 5.6 & 7.5 & 3.4 & 1.2 \\
Area $\left(\mathrm{km}^{2}\right)$ & 66.6 & 42.3 & 92.3 & 27.6 \\
\hline
\end{tabular}

$\mathrm{NDWI}_{56}=\frac{\text { band5 }- \text { band } 6}{\text { band5 }+ \text { band } 6}$

$N_{\mathrm{m}}=\sum_{i=1}^{n} \mathrm{GCP}_{i}$

and

$\mathrm{GCP}_{i}= \begin{cases}1 & \text { if } V_{\mathrm{GCP}}<V_{\text {Threshold }}<V_{\text {Soil }} \\ 0 & \text { else }\end{cases}$

where $N_{\mathrm{m}}$ is the number of correct GCPs located along the boundary pixels of classified water bodies when a trial threshold is used, $\mathrm{GCP}_{i}$ is the $i$ th GCP, $n$ is the number of GCPs used, $V_{\mathrm{GCP}}$ is the pixel value of the GCP, $V_{\text {Soil }}$ is the pixel value of pure soil, which is adjacent to the GCP, and $V_{\text {Threshold }}$ is the threshold value computed from the minimum value of the GCP pixels to their maximum value. Its interval was 0.0001 for NDWI images and single bands with reflectance, and 1 for single bands with DN values.

The function $V_{\mathrm{GCP}}<V_{\text {Threshold }}<V_{\text {Soil }}$ was applied to identify the threshold value for a single band, including both DN and spectral reflectance imagery. For NDWI images, the function $V_{\text {Soil }}<V_{\text {Threshold }}<V_{\mathrm{GCP}}$ was used. The threshold corresponding to the maximum $N_{\mathrm{m}}$ was selected as the optimized threshold for each independent data series. Then, the average selected threshold value for the 100 independent datasets was identified as the optimized threshold to distinguish water body pixels from remote sensing imagery.

3. The optimized threshold was used to classify water bodies for each image, including single bands with DN values, single bands with spectral reflectance, and the calculated NDWI images. The constraint function Pixel value $\leq$ optimized threshold was applied to classify water bodies for single-band images, whereas Pixel value $\geq$ the optimized threshold was applied to NDWI images.

4. Each dataset of threshold calibration points and threshold validation points was intersected with classified water body maps to verify the GCPs, which were correctly classified by each method. Both mean and 


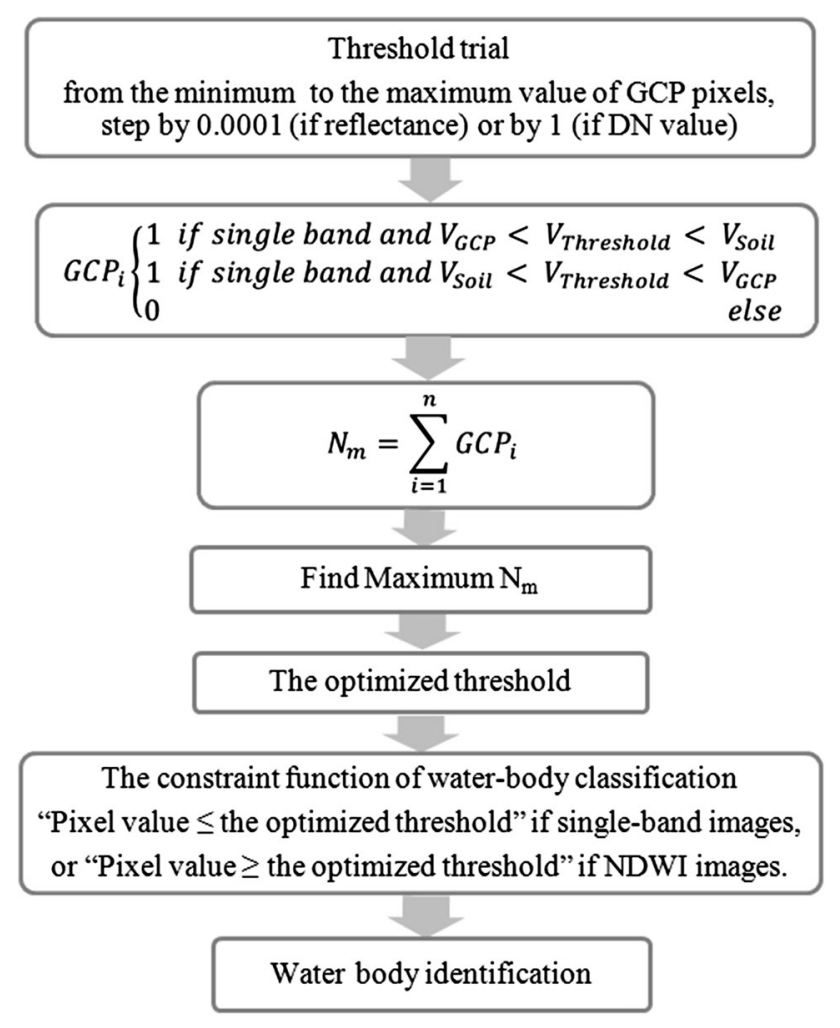

Fig. 2 The procedure of optimized threshold estimation and water body classification

standard deviation values for the 100 independent datasets were calculated. Finally, the performance of methods for identifying open water bodies from Landsat 8 OLI imagery was evaluated.

The optimized threshold estimation procedure and water body classification are shown in Fig. 2.

\section{Results}

\section{Calibration of the optimized threshold}

Randomly assigned GCP datasets were used to calibrate the optimized threshold for each image, including single bands with DN values and spectral reflectance, and the calculated NDWI images. A different optimized threshold was calibrated for each independent dataset using the threshold selection method described above.

The optimized thresholds of the independent datasets for each method, including the mean and the standard deviation, are shown in Fig. 3. The figure shows that the optimized threshold of NDWI35 was approximately -0.05 and -0.06 for reflectance images and DN value images, respectively. The standard deviations of the 100 independent datasets for reflectance images and DN value images (a)

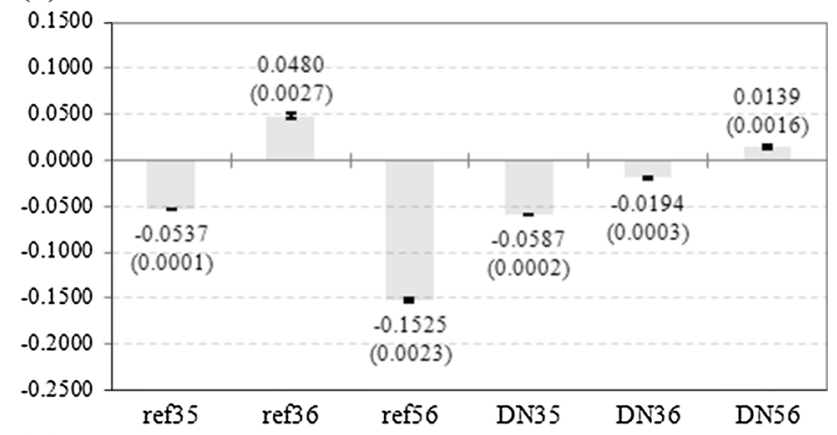

(b)

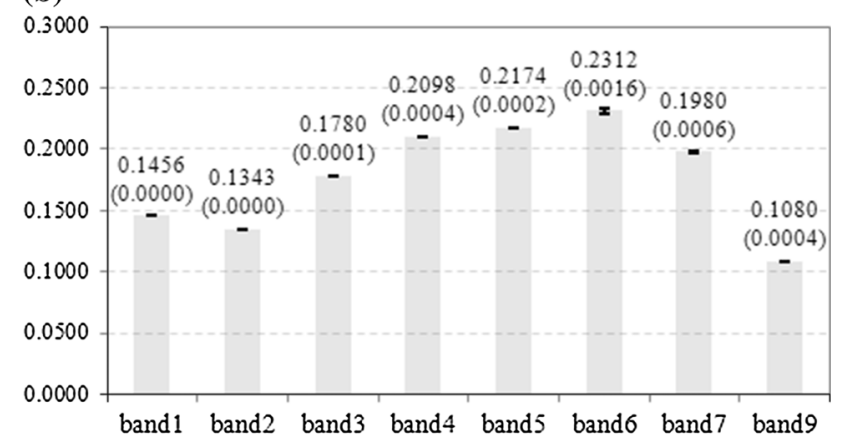

(c)

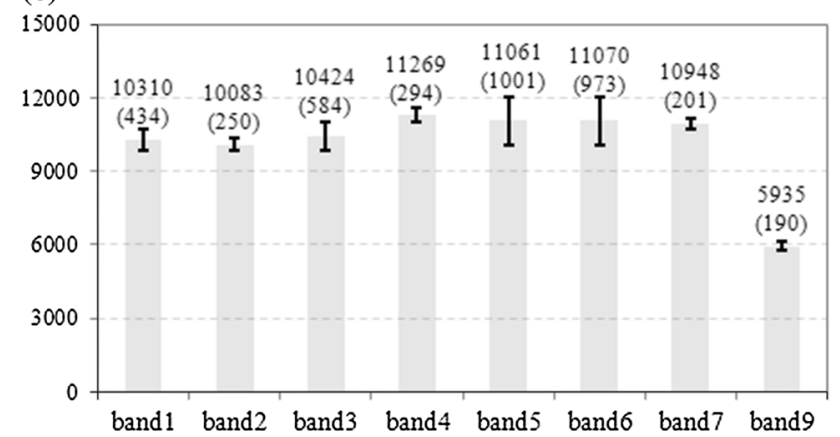

Fig. 3 Mean and stand deviation values of the optimized threshold for each method. a Shows NDWI methods; $\mathbf{b}$ and $\mathbf{c}$ are single-band methods with reflectance and DN values, respectively; "ref" and "DN" describe reflectance and DN values, respectively; "35," "36," and " 56 " describe NDWIs calculated by (Green - NIR)/(Green + NIR), (Green - MIR)/(Green + MIR), and (NIR - MIR)/(NIR + MIR), respectively

were 0.0001 and 0.0002 , respectively. These values were considerably lower than the mean optimized threshold, which indicated that the optimized threshold calibrated using the GCPs was stable for NDWI35 for both reflectance and DN value images. That is, the selection of randomly assigned GCP calibration datasets had little impact on the identification of the optimized threshold for NDWI35. The mean value of the optimized threshold for NDWI36 was approximately 0.05 and -0.02 for reflectance images and DN value images, respectively. The standard deviation of NDWI36 for DN value images was also much lower than the corresponding mean value. However, this value was comparatively high for the 
NDWI36 reflectance image. This the highest value of standard deviation in the NDWI images at 0.0027. This meant that the selection of GCP calibration datasets might affect the identification of the optimized threshold within the NDWI36 reflectance image. This was due in part to the fact that the NDWI36 was initially developed for DN value images (Xu 2006).

The mean value of the optimized threshold for single bands with reflectance varied from 0.1080 in band 9-0.2312 in band 6. The standard deviation of all single bands with reflectance was much lower than their mean values. This indicates that the optimized threshold was stable for single bands with reflectance. The DN values of the Landsat 8 OLI images varied from 1 to 65,535. The range was larger than that of Landsat Thematic Mapper/ Enhanced Thematic Mapper Plus (TM/ETM+) images, the values of which ranged from 1 to 255 .

The mean value of the optimized threshold for DN value images varied from 5935 in band 9-11,269 in band 4. The standard deviation of single bands with $\mathrm{DN}$ values was relatively high compared to the corresponding mean value. The standard error of the mean for single bands with DN values was larger than that for single bands with reflectance. This indicates that the optimized threshold was more stable for single bands with reflectance than for those with DN values. The selection of randomly assigned GCP calibration datasets might have had some effect on optimized threshold identification for single bands with DN values.

\section{Performance of methods on calibration and validation datasets}

The optimized threshold was used to classify water bodies for each image. Each dataset of the threshold calibration and threshold validation points intersected with the classified water body maps to verify the correct GCPs. The calibration and validation methods were then evaluated for each of the 100 independent datasets.

The performance of the methods for GCP calibration is shown in Fig. 4. In general, almost all methods captured more than $60 \%$ of the GCPs in identifying water bodies in remote sensing images, except for the single band 2 with DN values. The NDWI35 image with reflectance yielded the best performance of the methods tested, with a mean value of correct identification of GCPs greater than $95 \%$. It also showed the most stable performance for the NDWI35 reflectance image in the calibration because both the range and the standard deviation that it obtained for the 100 independent datasets were the lowest of the methods. Moreover, it exhibited better performance for the NDWI35 image with DN values, and the single band 6 with reflectance and DN values, which means that the values for more than $90 \%$ of the GCPs were correct in the calibration.
The effects of selecting randomly assigned GCP calibration datasets on the performance for the NDWIs methods, which were in the lower range and standard deviation for the 100 independent datasets, were less pronounced than those for the other methods that were tested. The performance of the NDWI35 was slightly better for the reflectance image than the DN value image, whereas the performance of the NDWI36 was slightly better for the DN value image than the reflectance image. This result was consistent with initial definitions where the NDWI35 was first developed for images with reflectance, whereas the NDWI36 was first defined by images with DN values. The NDWI56 image with reflectance recorded nearly $90 \%$ correct GCPs in the calibration, but with DN values that only captured approximately $65 \%$ correct GCPs. The result was also consistent with its initial definition, whereby the NDWI56 was developed for images with reflectance.

The single-band method in general showed little difference between the reflectance value image and the DN value image. The performance of methods that used band 6 and band 7 was clearly better than that of other single-band methods. The single-band method also showed relatively satisfactory performance for band 5 and band 9. However, the single-band method for bands 1-3 in calibration captured fewer than $70 \%$ of the correct GCPs.

Figure 5 shows the performance of the methods for randomly assigned GCP validation datasets, which included the 100 independent datasets. Overall, the performance of each method in the validation phase corresponded to its results for the calibration phase. However, the range and the standard deviation of the 100 independent datasets were clearly larger in the validation than in the calibration. The NDWI35 image with reflectance exhibited the best performance in validation as well.

More than $90 \%$ of the correct GCPs for the NDWI35 image in each independent validation dataset were identified, with a mean value greater than $95 \%$. The range and the standard deviation of the 100 independent datasets for the NDWI35 image were the lowest among the methods in validation, which indicated that the NDWI35 method with reflectance was the most stable method for identifying water bodies from remote sensing images.

The mean DN validation values of correct GCPs were more than $90 \%$ for the NDWI35 image and the singleband 6 with reflectance and DN values. The selection of randomly assigned GCP validation datasets had relatively little effect on the performance of these three methods than on the other methods tested because the former had a lower range and standard deviation for the 100 independent datasets. This indicates that these three methods are superior at identifying water bodies from remote sensing imagery than the other methods that were tested. The 
Fig. 4 The performance of different methods in calibration datasets of ground control points ("ref" and "DN" describe reflectance and DN values, respectively; " 35 ," "36," and " 56 " describe NDWIs calculated by (Green - NIR)/

(Green + NIR),

(Green - MIR)/

$($ Green + MIR), and (NIR - MIR)/(NIR + MIR), respectively; "b1" to "b9" represent band 1 to band 9 , respectively)
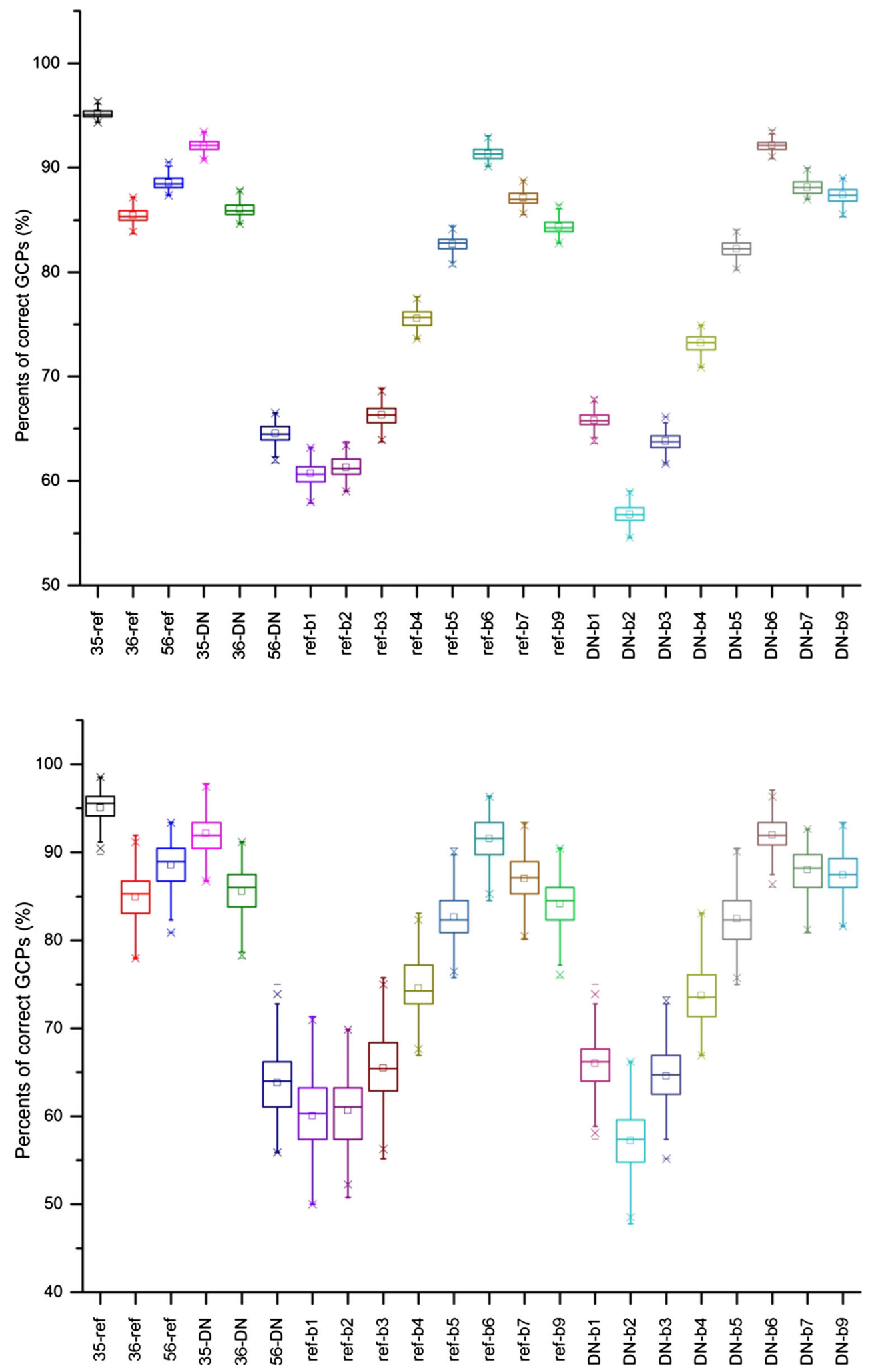

Fig. 5 The performance of different methods in validation datasets of ground control points ("ref" and "DN" describe reflectance and DN values, respectively; " 35 ," "36," and " 56 " describe NDWIs calculated by (Green - NIR)/

$($ Green + NIR $)$,

(Green - MIR)/

(Green + MIR), and (NIR - MIR)/(NIR + MIR), respectively; "b1" to "b9" represent band $1-$ band 9 , respectively) performance of the NDWI35 was slightly better for the reflectance image than the DN value image, whereas the performance of the NDWI36 was slightly better for the DN value image than the reflectance image in the validation. This result confirmed its initial definition, whereby the NDWI35 was developed for images with reflectance, 
whereas the NDWI36 was defined for images with DN values. The mean value of correct GCPs was greater than $90 \%$ for the NDWI56 image with reflectance, but was smaller than $65 \%$ for the image with DN values. This was because the NDWI56 was initially developed for images with reflectance, and not for images with $\mathrm{DN}$ values.

The single-band method exhibited little difference between the reflectance value image and the $\mathrm{DN}$ value image, which was the same as that in the calibration. In general, the single-band method yielded the best performance in band 6 for validation, with a mean value of greater than $90 \%$ of correct GCPs. It exhibited better performance from band 5 to band 9 , which captured more than $80 \%$ of the correct GCPs in the validation. However, the single-band method for bands 1-3 captured fewer than $70 \%$ of the correct GCPs in the validation.

The performance of the methods for calibration and validation shows that the NDWI35 image with reflectance was the best method for identifying water bodies from remote sensing imagery. The difference in performance between reflectance and DN values of NDWI images was consistent with their initial definitions. For example, NDWI35, NDWI36, and NDWI56 were initially developed for reflectance, DN value, and reflectance, respectively. The results of calibration and validation in this study showed that the NDWI35 and the NDWI56 performed better with reflectance images than DN value images, whereas NDWI36 was better for DN value images.

\section{The difference between the pixels of pure water and the soil adjoining the GCPs}

The mean values and the ratio of pure water pixels to the pixels of soil adjoining the GCPs in each single band, including both reflectance images and DN values images, are shown in Fig. 6. The mean value of pure water pixels adjacent to GCPs was less than 0.01 from images for reflectance from band 5 to band 9, with the lowest value being 0.0037 for band 9 . The mean value of pure water pixels adjacent to GCPs was obviously larger from band 1 to band 4 than that from band 5 to band 9 . For the bands from band 1 to band 4, the value was from 0.03 in band 4 to 0.09 for band 3 .

The mean value of pixels representing pure soil ranged from 0.16 in band 9 to 0.32 in band 6 . The difference between pure water pixels and pure soil pixels was clearly greater in band 5 than in band 9, with the largest difference in band 6 , where the ratio of pure water to pure soil was approximately $62: 1$. This means that the values of the pure soil pixels were as much as 62 times those of the pure water pixels adjacent to the GCPs.

For single-band images with DN values, the result was similar to that for images with reflectance. The mean value of pure water pixels in band 5 to band 9 images was clearly lower than that in images from band 1 to band 4 . That of pure soil pixels was greater in band 4 to band 7 , but recorded the lowest value in band 9 with a mean value of 6800. The difference between pure water and pure soil pixels was greater in band 4-band 7, with the largest difference in band 6 , where the ratio of pure water to pure soil was approximately $3: 1$. In other words, the difference in the DN values of pure soil and water was greater in band 6 than in any other band.

The largest difference in pixel values occurred between pure water and pure soil in band 6 for both reflectance and DN values images, while it showed a relatively small difference in bands $1-3$. This confirmed satisfactory results for the performance of the single-band method in identifying water bodies from images, with the single band 6 method showing the best performance. Descriptions of the difference in pixel reflectance values between pure water pixels and pure soil pixels for the typical band 2 and band 6 are shown in Fig. 7. The figure shows that the values of pure water pixels, which are adjacent to the GCPs, were less than 0.05 in band 6 . The values of pure water pixels were lower than those of pure soil pixels in this band-the largest pure water pixel value (0.050) was lower than the smallest pure soil pixel (0.241)—with an optimized threshold value of 0.2312 .

Single-band 2 showed some overlap between pure water and pure soil pixel values. This was similar to other bands, which are not shown. Figure 8 shows the difference in DN values between pure water pixels and pure soil pixels for the typical band 2 and band 6 . The results were similar to those of reflectance value images. In general, band 6 is the most suitable for identifying water bodies from single-band images.

\section{Evaluation of the frequency histogram method in the classification of water bodies}

As mentioned above, the frequency histogram method, also called the density slicing method (Ryu et al. 2002), is among the most popular and effective methods for selecting an appropriate threshold when identifying water bodies from remote sensing imagery. The characteristics of the spectral reflectance of water and soils must be understood and considered before applying the frequency histogram method.

In general, the distribution of histograms of the NDWI images was bimodal or trimodal. Two peaks representing water and soil pixels were found in all histograms. The difference between the trough values of these two peaks was chosen as the threshold. However, the distribution of the histograms was unimodal for single-band images, where an appropriate threshold was difficult to identify. 
Fig. 6 Mean values of pure water and pure soil pixels adjacent to ground control points in each single band

Fig. 7 Pixel reflectance values of pure water and pure soil adjacent to ground control points in band 2 and band 6

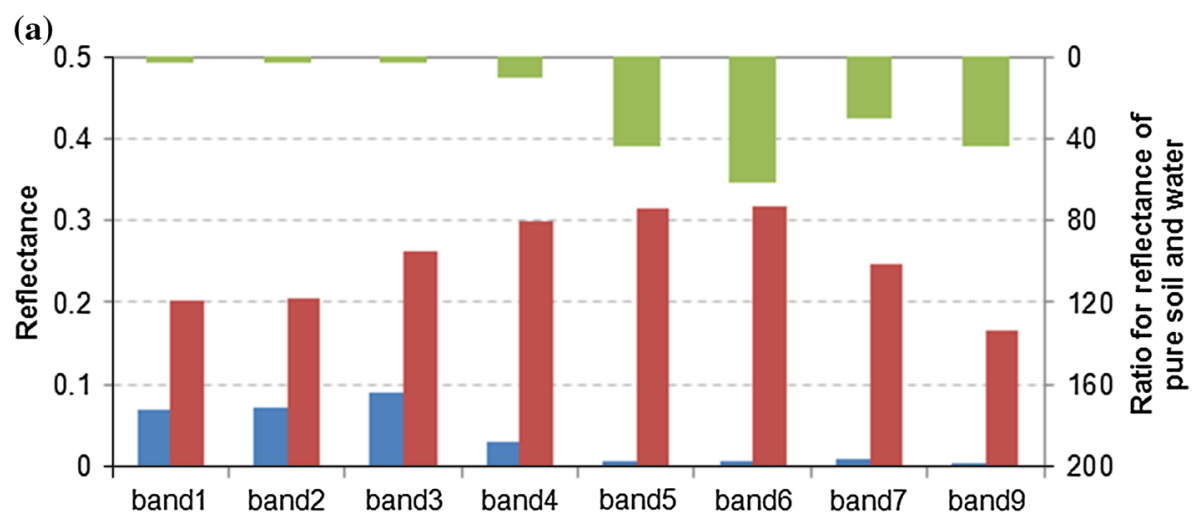

(b)
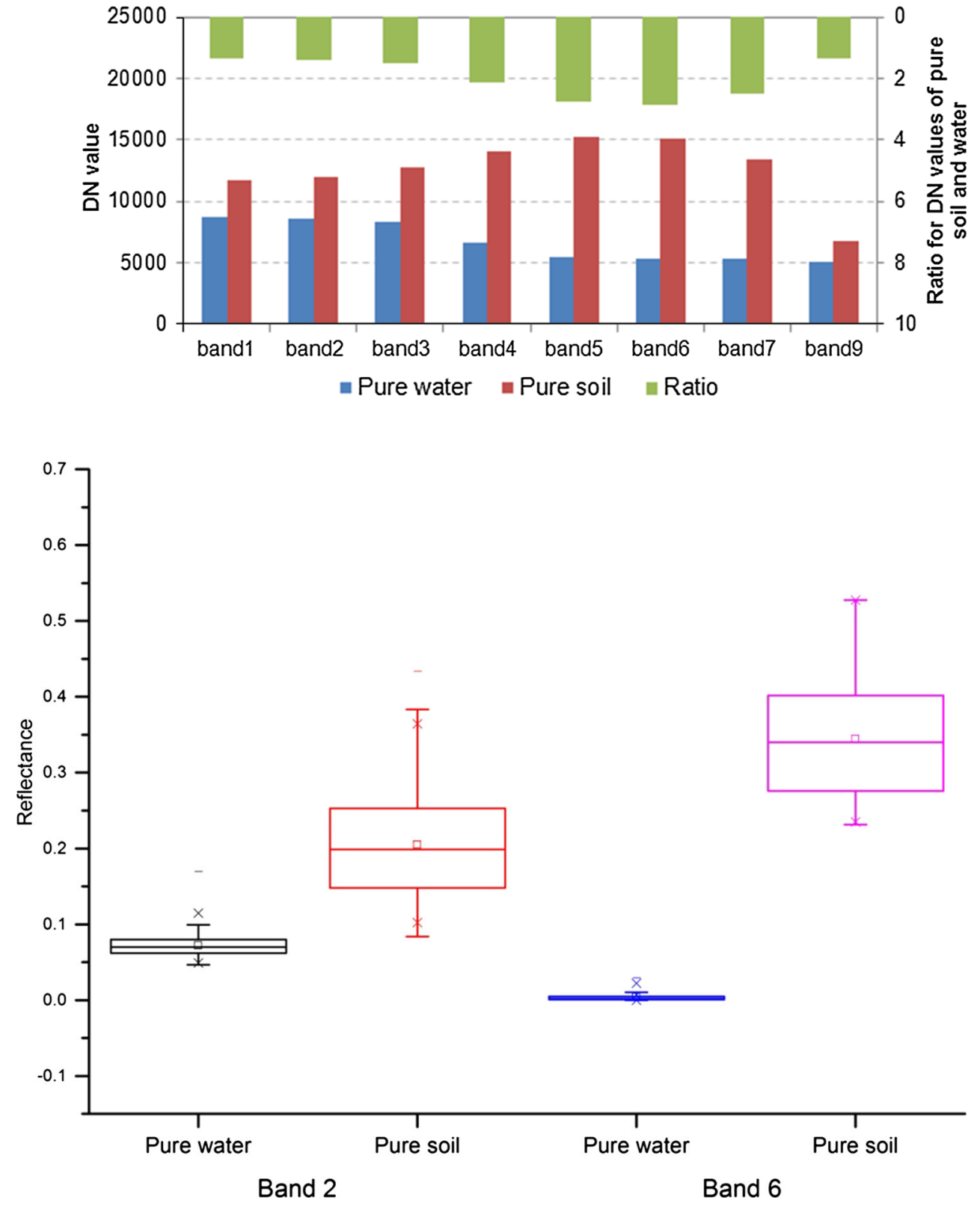
Fig. 8 Pixel DN values of pure water and pure soil adjacent to ground control points in band 2 and band 6

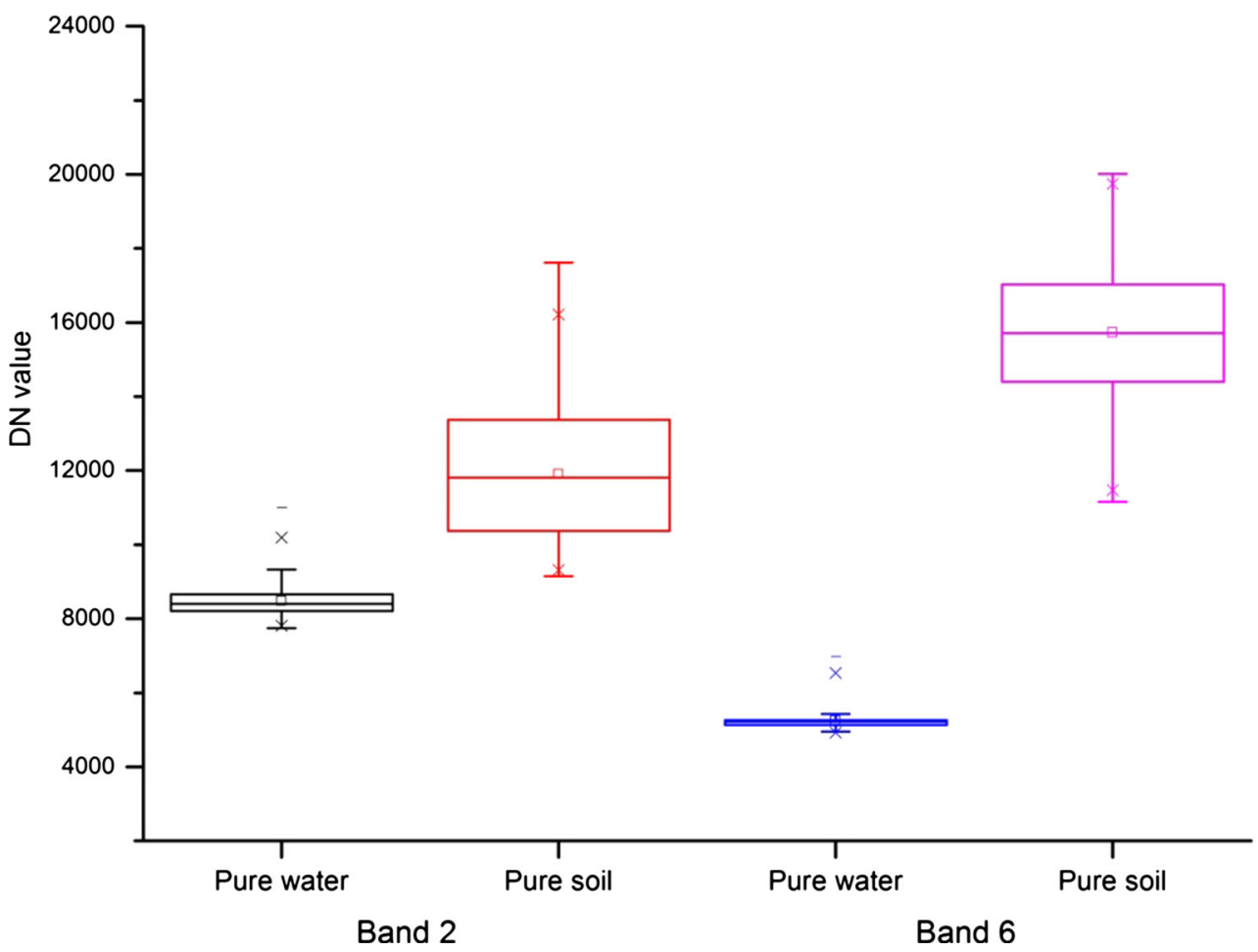

Table 2 NDWI imagery thresholds identified by the frequency histogram method

\begin{tabular}{llllll}
\hline & \multicolumn{2}{l}{ The whole image } & & \multicolumn{2}{l}{ A part of the image } \\
\cline { 2 - 3 } \cline { 6 - 6 } & Reflectance & DN & & Reflectance & DN \\
\hline NDWI35 & 0.01 & -0.01 & & -0.05 & -0.04 \\
NDWI36 & 0.21 & 0.06 & & 0.16 & 0.04 \\
NDWI56 & 0.18 & 0.01 & & 0.11 & 0 \\
\hline
\end{tabular}

The frequency histogram of each NDWI image was used to obtain threshold values to distinguish water bodies from other features of the landscape. These threshold values are shown in Table 2.

The water area in the image is relatively smaller than the non-water areas. Therefore, the part of the image where the lake area accounted for a larger proportion of the entire area was selected to identify thresholds by the frequency histogram method. This part of the NDWI image enhanced water body classification performance using the frequency histogram method because the thresholds identified in this part were invariably closer to the optimized thresholds calibrated by GCPs (Fig. 3) than those identified for the entire image. This implies that the extraction of a part of the NDWI image, where the area occupied by the water body accounts for a large proportion of the entire area, is necessary for accurate water body classification when the frequency histogram method is used. With regard to different NDWI indices, the threshold identified by the

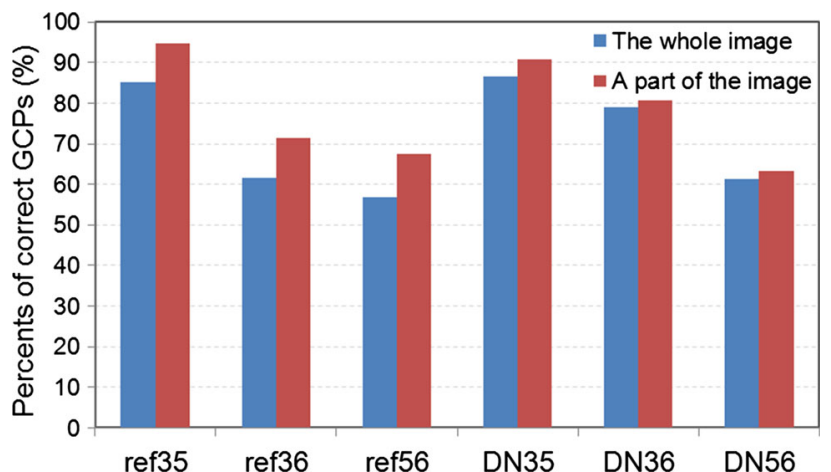

Fig. 9 The performance of the frequency histogram method on ground control points ("ref" and "DN" describe reflectance and DN values respectively; "35," "36," and "56" describe NDWIs calculated by $($ Green - NIR $) /($ Green + NIR $),($ Green - MIR)/(Green + MIR), and (NIR - MIR)/(NIR + MIR), respectively)

frequency histogram method was closest to the optimized thresholds in the NDWI35 images.

Water bodies were identified from NDWI images based on thresholds selected by the frequency histogram method. The performance of these classifications was evaluated by GCPs, and the results are shown in Fig. 9. In general, the water body classification performance using part of an NDWI image was superior to that using the entire corresponding image. This also explains the necessity of extracting an appropriate part of the NDWI image for water body classification when the frequency histogram method is used. For part of the image, the frequency histogram 
method for the NDWI35 images with for reflectance and DN values captured more than $90 \%$ of the correct GCPs in the classification of water bodies. The frequency histogram for the NDWI36 image with DN values captured more than $80 \%$ of the correct GCPs. However, the frequency histogram for the NDWI56 recorded an accuracy of lower than $70 \%$. In applications of the frequency histogram method, the NDWI35 was the most reliable NDWI index for the classification of water bodies.

\section{Discussion}

\section{Threshold calibration}

For NDWI methods, Gao (1996) suggested that the NDWI56 value for vegetation water was positive, whereas that for bare soils was negative. This threshold of zero has been applied by Ma et al. (2007) to identify lake areas. However, it was evaluated as -0.1 by field control points in detecting water in the Sahara-Sahel transition zone (Campos et al. 2012). That result has been confirmed in this study.

The optimized threshold, calibrated and validated by 737 GCPs, was $-0.1525 \pm 0.0023$ in our study area. The threshold for the NDWI35 and the NDWI36 was initially proposed to be zero (McFeeters 1996; Xu 2006). However, as mentioned above, threshold adjustment can effect a more accurate delineation of water bodies in specific situations (Ji et al. 2009). Alterable thresholds have been employed in the past to glean information regarding water bodies in different regions based on the maximum interclass variance method, the frequency histogram method, and the field control points' calibration method. The first two methods are image threshold segmentation methods. Table 2 lists examples of thresholds identified in different regions when detecting water areas from Landsat TM/ ETM+ images with reflectance values. Image threshold segmentation methods are useful for identifying water bodies from remote sensing images when field data are lacking, but field datasets are needed to confirm their classifications. Therefore, field control point calibration methods provide more accurate water classification results they can be confirmed. These results showed that the optimized threshold was -0.05 at both the Sahara-Sahel transition zone (Campos et al. 2012) and the northern Tibetan plateau in this study.

In general, the distribution of the histograms is bimodal or trimodal for NDWI images of areas with large water bodies. The histogram method identified a threshold between the two peaks of water and non-water pixels. However, the distribution of the histograms was always unimodal for single-band images. It is difficult to identify an appropriate threshold from a histogram with a unimodal distribution. Therefore, the NDWI methods were more suitable for water body classification than single-band methods when the frequency histogram method was used.

\section{Performance differences between reflectance and DN value images}

Spectral reflectance images are recommended for quantitative remote sensing of land surfaces (Liang 2003). DN value images should be converted into physical quantities to estimate land surface variables. However, DN value images show some ability for identifying water bodies from NDWI images calculated from DN values, especially for the NDWI35 and the NDWI 36 indices. This is possibly because $\mathrm{DN}$ value images are strongly correlated with spectral reflectance. The range $(1-65,555)$ of $\mathrm{DN}$ values in Landsat 8 imagery was much larger than that in previous versions of imagery (1-255), which might also account for the ability of this method to identify water bodies.

The performance of NDWI images for reflectance and DN values was consistent with their initial definitions: the NDWI35, and NDWI56 and NDWI36 were initially developed for reflectance and DN values, respectively. The results of calibration and validation in this study showed that the performance of the NDWI35 and the NDWI56 was better for reflectance images than DN value images, and that of the NDWI36 was better for DN value images. Therefore, we conclude that the NDWI indices calculated by their initial definitions can produce more reasonable results in the classification of water bodies.

\section{Assessment of water body classification by different methods}

Different methods were calibrated and validated by randomly assigned GCP datasets, which included 100 independent datasets. Results showed that the NDWI35 image with reflectance was the best method for identifying water bodies in remote sensing images and captured more than $95 \%$ correct GCPs. Threshold calibration based on field datasets showed that the optimized threshold was -0.05 at both the Sahara-Sahel transition zone (Campos et al. 2012) and the northern Tibetan plateau. We believe the optimized threshold of the NDWI35 should be set to -0.05 instead of zero, which was used in many studies.

However, more optimized thresholds in other typical regions need to be calibrated and validated as well. Further, three other methods-NDWI35 images with DN values, single-band 6 with reflectance, and with DN valuesyielded relatively satisfactory performance in our experiment, which means that they captured more than $90 \%$ correct GCPs. The selection of randomly assigned GCP 
datasets had relatively little effect on the performance of these four methods than on other methods because the former had lower range and lower standard deviation values for the 100 independent datasets. The performance of the NDWI56 was generally poorer than that of the NDWI35 and the NDWI36, especially for indices calculated from DN values. This could have been the case because the NDWI35 and the NDWI36 were initially developed for identifying open water bodies, whereas the NDWI56 was developed for vegetation water. This indicates that the NDWI35 and the NDWI36 were the preferred NDWI indices when detecting open water bodies from remote sensing imagery.

\section{Conclusions}

In this paper, we used 737 GCPs to evaluate methods for identifying open water bodies from Landsat 8 OLI imagery. Our conclusions are as follows.

The NDWI35 image with reflectance was the best method for identifying water bodies in remote sensing images, capturing over $95 \%$ correct GCPs in the studied region. When the frequency histogram method was used, the NDWI methods were more suitable for water body classification than single-band methods. The NDWI35 exhibited the best performance. Therefore, we recommend the NDWI35 for the classification of water bodies when the frequency histogram method is used.

The NDWI indices calculated by their initial definitions can produce more reasonable results in the classification of water bodies. The NDWI35 and the NDWI56 performed better for reflectance images than DN value images, whereas the NDWI36 yielded better results for DN value images.

The optimized threshold of the NDWI35 might be better set to -0.05 instead of 0 , which is used in many studies. At the same time, more optimized thresholds in other typical regions need to be calibrated and validated if data are available.

Acknowledgments This study was supported and funded by National Natural Science Foundation of China (Grants Nos. 41190080, 41201035 and 41371058). We would also like to thank Liguang Jiang, Junbo Wang, Jianting Ju, and Yong Wang for field assistance.

\section{References}

Beyer HL (2004) Hawth's analysis tools for ArcGIS. http://www. spatialecology.com/htools

Bishop MP, Olsenholler JA, Shroder JF, Barry RG, Raup BH, Bush ABG, Copland L, Dwyer JL, Fountain AG, Haeberli W, Kääb A, Paul F, Hall DK, Kargel JS, Molnia BF, Trabant DC, Wessels R (2004) Global land Ice measurements from space (GLIMS): remote sensing and GIS investigations of the Earth's Cryosphere. Geocarto Int 19(2):57-84

Braud DH, Feng W (1998) Semi-automated construction of the Louisiana coastline digital land/water boundary using Landsat Thematic Mapper satellite imagery. Louisiana Applied Oil Spill Research and Development Program, OSRAPD Technical Report Series 97-002

Campos JC, Sillero N, Brito JC (2012) Normalized difference water indexes have dissimilar performances in detecting seasonal and permanent water in the Sahara-Sahel transition zone. J Hydrol 464-465:438-446

de Alwis DA, Easton ZM, Dahlke HE, Philpot WD, Steenhuis TS (2007) Unsupervised classification of saturated areas using a time series of remotely sensed images. Hydrol Earth Syst Sci 11(5):1609-1620

Dong GC, Yi CL, Chen LJ (2010) An introduction to the physical geography of the Qiangtang Plateau: a frontier for future geoscience research on the Tibetan Plateau. Phys Geogr 31(6):475-492

Dozier J (1984) Snow-reflectance from Landsat-4 thematic mapper. IEEE T Geosci Remote GE 22(3):323-328

Dozier J (1989) Spectral signature of alpine snow cover from the Landsat Thematic Mapper. Remote Sens Environ 28:9-22

Eastman JR (2001) Introduction to remote sensing and image processing. Guide to GIS and image processing, vol 1. Clark Labs, Worcester, MA, pp 27-28

Frazier PS, Page KL (2000) Water body detection and delineation with Landsat TM data. Photogramm Eng Remote Sens 66(12):1461-1467

Gao BC (1996) NDWI-A normalized difference water index for remote sensing of vegetation liquid water from space. Remote Sens Environ 58(3):257-266

Hepner GF, Logan T, Ritter N, Bryant N (1990) Artificial neural network classification using a minimal training set-comparison to conventional supervised classification. Photogramm Eng Remote Sens 56:469-473

Ji L, Zhang L, Wylie B (2009) Analysis of dynamic thresholds for the normalized difference water index. Photogramm Eng Remote Sens 75(11):1307-1317

Lacaux JP, Tourre YM, Vignolles C, Ndione JA, Lafaye M (2007) Classification of ponds from high-spatial resolution remote sensing: application to Rift Valley Fever epidemics in Senegal. Remote Sens Environ 106(1):66-74

Leauthaud C, Belaud G, Duvail S, Moussa R, Grünberger O, Albergel $\mathrm{J}$ (2013) Characterizing floods in the poorly gauged wetlands of the Tana River Delta, Kenya, using a water balance model and satellite data. Hydrol Earth Syst Sci 17:3059-3075

Li JL, Sheng YW (2012) An automated scheme for glacial lake dynamics mapping using Landsat imagery and digital elevation models: a case study in the Himalayas. Int J Remote Sens 33(16):5194-5213

Li WB, Du ZQ, Ling F, Zhou DB, Wang HL, Gui YM, Sun BY, Zhang XM (2013) A comparison of land surface water mapping using the normalized difference water index from TM, ETM+ and ALI. Remote Sens 5:5530-5549

Liang SL (2003) Quantitative remote sensing of land surfaces. Wiley, New Jersey, pp 2-3

Lu SL, Ouyang NL, Wu BF, Wei YP, Tesemma Z (2013) Lake water volume calculation with time series remote-sensing images. Int $\mathrm{J}$ Remote Sens 34(22):7962-7973

Ma M, Wang X, Veroustraete F, Dong L (2007) Change in area of Ebinur Lake during the 1998-2005 period. Int J Remote Sens 28(24):5523-5533

Manavalan P, Sathyanath P, Rajegowda GL (1993) Digital image analysis techniques to estimate waterspread for capacity evaluations of reservoirs. Photogramm Eng Remote Sens 59(9):1389-1395 
McFeeters SK (1996) The use of the Normalized Difference Water Index (NDWI) in the delineation of open water features. Int $\mathbf{J}$ Remote Sens 17:1425-1432

Moller-Jensen L (1990) Knowledge-based classification of an urban area using texture and context information in Landsat TM imagery. Photogramm Eng Remote Sens 56:475-479

Okin GS, Roberts DA, Murray B, Okin WJ (2001) Practical limits on hyperspectral vegetation discrimination in arid and semiarid environments. Remote Sens Environ 77:212-225

Paola JD, Schowengerdt RA (1995) A detailed comparison of backpropagation neural network and maximum-likelihood classifiers for urban land use classification. IEEE T Geosci Remote 33(4):981-996

Raup B, Kääb A, Kargel JS, Bishop MP, Hamilton G, Lee E, Paul F, Rau F, Soltesz D, Khalsa SJS, Beedle M, Helm C (2007) Remote sensing and GIS technology in the global land ice measurements from space (GLIMS) project. Comput Geosci 33:104-125

Ryu JH, Won JS, Min KD (2002) Waterline extraction from Landsat TM data in a tidal flat: a case study in Gomso Bay, Korea. Remote Sens Environ 83:442-456

Smith RD (2012a) Introduction to remote sensing of the environment. http://www.microimages.com/getstart/pdf/introrse.pdf

Smith RD (2012b) Introduction to hyperspectral Imaging. http:// www.microimages.com/documentation/Tutorials/hyprspec.pdf

Sun FD, Sun WX, Chen J, Gong P (2012) Comparison and improvement of methods for identifying waterbodies in remotely sensed imagery. Int J Remote Sens 33(21):6854-6875

$\mathrm{Xu} \mathrm{H}$ (2006) Modification of normalised difference water index (NDWI) to enhance open water features in remotely sensed imagery. Int J Remote Sens 27:3025-3033 